\title{
LA RELATION ENTRE LA QUALITE DE L'INFORMATION COMPTABLE ET L'ORGANISATION DU SERVICE QUI LA PRODUIT : UNE ETUDE EMPIRIQUE DANS LE CONTEXTE AGRICOLE MAROCAIN
}

\section{THE RELATIONSHIP BETWEEN THE QUALITY OF ACCOUNTING INFORMATION AND THE ORGANIZATION OF THE SERVICE THAT PRODUCES IT: AN EMPIRICAL STUDY IN THE MOROCCAN AGRICULTURAL CONTEXT}

\author{
AZHARI Amine ${ }^{1}$, BOUAZIZ Si Mohamed ${ }^{2}$ \\ ${ }^{1}$ Faculté des Sciences Juridiques, Économiques et Sociales, Agadir, MAROC \\ ${ }^{2}$ Faculté des Sciences Juridiques, Économiques et Sociales, Agadir, MAROC
}

\begin{abstract}
Résumé : Le présent papier a pour objectif de mettre en lumière comment l'organisation du service comptable impact la qualité de l'information comptable des entreprises agricoles de la région du SoussMassa. Pour préserver une harmonie entre l'objet de notre recherche et le chemin méthodologique parcouru pour l'appréhender, nous avons élaboré un modèle conceptuel composé de treize variables réparties sur trois niveaux issus des politiques de gouvernance de l'entreprise. Partant d'un paradigme post-positiviste que nous avons inscrit dans un raisonnement hypothético-déductif, le passage du socle théorique au versant empirique de l'étude s'est fait à travers une investigation par modélisation structurelle de troisième ordre à variables latentes estimée selon l'approche des indicateurs répétés suivant un modèle de type II (réflectif-formatif).

Ce modèle qui a été soumis à l'épreuve sur un échantillon de 213 observations, a permis de remettre en cause l'hypothèse globale du travail, une conclusion d'autant plus étonnante, dans la mesure où elle s'oppose aux aboutissements développés par de Michailesco (1998) et de Mahmoud (2012) selon lesquels, un des aspects supposés influencer la qualité de l'information comptable est les caractéristiques internes du service comptable de l'entreprise au sens ou la qualification du comptable, l'organisation et la digitalisation du service comptable devraient favoriser la pertinence de l'information comptable.
\end{abstract}

Mots clés : Qualité de l'information comptable ; service comptable ; Entreprise agricole ; Modélisation par équations structurelles.

\footnotetext{
Abstract: The objective of this paper is to highlight how the organization of the accounting department impacts the quality of accounting information of agricultural enterprises in the Souss-Massa region. In order to maintain harmony between the object of our research and the methodological path taken to understand it, we have developed a conceptual model composed of thirteen variables distributed over
} 
three levels stemming from the company's governance policies. Starting from a post-positivist paradigm that we have inscribed in a hypothetico-deductive reasoning, the passage from the theoretical base to the empirical side of the study was done through an investigation by structural modeling of third order with latent variables estimated according to the approach of repeated indicators following a type II model (reflective-formative).

This model, which was tested on a sample of 213 observations, allowed to question the global hypothesis of the work, a conclusion all the more surprising, insofar as it opposes the results developed by Michailesco (1998) and Mahmoud (2012) according to which, one of the aspects supposed to influence the quality of accounting information is the internal characteristics of the accounting department of the company in the sense that the qualification of the accountant, the organization and the digitalization of the accounting department should promote the relevance of the accounting information

Key words: Quality of accounting information; accounting department; agricultural firm; structural equation modeling.

\section{Introduction}

La comptabilité est considérée comme ayant joué un rôle très positif dans la création d'un domaine organisationnel gérable. Un régime de visibilité et de calcul économique a permis d'assurer une légitimité de l'entreprise qui, grâce à ce régime, elle se voit aujourd'hui en taille d'atteindre ses objectifs (Hopwood, 1987). La reddition des comptes permet une meilleure traduction des actes de l'entreprise en incorporant les motifs économiques dans des faits économiques légitimes et acceptés (ho, 1988). Pourtant, au gré des incommodités que présente l'environnement, il est pris en acte que la qualité de l'information rapportée dans les états de synthèses varie d'une entreprise à l'autre (Michaïlesco, 1998). Les auteurs qui se sont intéressés à expliquer la divergence des comportements observés chez les dirigeants des entreprises concernant leurs pratiques comptables, ont examinés les déterminants susceptibles d'influencer d'abord, les décisions prises en matière de publication, ensuite la qualité de l'information contenue dans ses publications et qui est destinées aux parties prenantes de l'entreprise (Miles et Miles, 2019).

La rétention ou la divulgation de l'information financière sont utilisées comme moyen d'assurer la légitimité du dirigeant de l'entreprise à l'interne comme à l'externe. À vrai dire, les dirigeants ont tendance de tisser des relations érigées sur une confiance échangée dans le cadre des groupes de travail afin de stimuler l'interdépendance des individus (Courpasson, 1997). L'implication des individus dans la stratégie de l'entreprise, suite à leurs interdépendances, induit une amélioration de la compétitivité et induit une auto surveillance des individus.

Dès lors, en plus de son rôle stratégique, la communication financière s'autorise à agir sur le plan tactique pour témoigner la légitimité interne du dirigeant. Bien entendu que l'information financière de l'entreprise a été préparée par le dirigeant, elle est réputée légitime attendu qu'elle découle d'une stratégie qui provient d'un travail de groupe. Bien que les études sur la théorie de la contingence n'aient pas toujours été cohérentes, et que l'approche soit limitée en termes de capacité à saisir les modèles et 
les dynamiques, la théorie de la contingence a néanmoins fourni au cours des trois dernières décennies un cadre théorique pratique pour de nombreuses études sur la structure et le comportement des organisations (Chenhall, 2003 ; 2007). En effet, la qualité de l'information comptable est considérablement affectée par les caractéristiques du service chargé de sa production et sa diffusion (Lavigne 2002 ; Chapelier, 1994 et Lacombe-Saboly, 1994).

L'étude de la littérature comptable traitant la qualité de l'information comptable nous a permis de dégager les principaux facteurs susceptibles d'affecter la qualité de l'information liées aux caractéristiques du service comptable de l'entreprise, au même titre que Gibbins. Dans le présent papier, nous discutons de tous ces déterminants que nous avons pu identifier et qui seront à la base des variables que nous utiliserons plus tard, après traitement, pour l'établissement de notre modèle hypothétique.

Partant des faits précédemment évoqués, nous précisons notre problématique par une question centrale comme il a été préconisé par Wacheux (1996). C'est ainsi que nos propos visent à savoir : Quelle est la relation entre la qualité de l'information comptable et l'organisation du service qui la produit ? Une question d'une importance singulière et novatrice dont très peu de réflexions et de recherches académiques ont accordé une attention.

Notre papier est organisé de la manière suivante. Le premier point est consacré à l'exposé d'une revue critique de la littérature conceptuelle et théorique se rapportant à la liaison entre les caractéristiques du service comptable de l'entreprise et la qualité de l'information comptable qu'il produit. Le deuxième point présente succinctement notre terrain de recherche, soit les entreprises agricoles issues de la région Souss-Massa ainsi que la démarche méthodologique prônée ayant servi à franchir les obstacles d'opérationnalisation de notre cadre conceptuel. Dans le troisième point, nous esquissons les résultats de l'étude avant de présenter, au niveau de la conclusion, une synthèse des implications ainsi que les voies futures sur lesquelles s'ouvre notre réflexion.

\section{Revue des facteurs incorporés aux contingences internes de l'entreprise}

Nous consacrons ce paragraphe à l'inventaire des variables liées aux caractéristiques du service comptable de l'entreprise susceptible d'avoir un impact sur la qualité de l'information divulguée. De nombreuses études empiriques (Alotaibi et Hussainey, 2016 ; Ortas et al., 2015 ; Alves et al., 2012 ; Liu et Anbumozhi, 2009 ; Raffounier, 2007 ; Barako et al., 2006 ; Michaïlesco, 1999 ; Michaïlesco, 1998) dans différents contextes ont partagé des variables de contingence qui convergent généralement sur :

- Morphologie de service comptable ;

- Degré d'informatisation de service comptable ;

- $\quad$ Profil de comptable et ; 


\subsection{L'effet de la morphologie du service comptable d'une entreprise sur la qualité de l'information comptable}

À travers la variable morphologie de service, nous tentons d'évaluer l'impact que peut avoir l'organisation de l'entité chargée de la production comptable sur la qualité de l'information comptable. C'est ainsi qu'on doit passer par la déclinaison de la variable en indicateurs statistiques pertinents que nous avons pu repérer auparavant dans la revue de la littérature (Nyengue Edimo, 2006).

Nous retenons donc, les deux indicateurs (Nombre d'employés du service comptable et Nombre de niveaux hiérarchiques du service comptable) comme bloc de mesure de la variable (Morphologie du service). L'agglomération des deux indicateurs renseigne implicitement sur la taille du service comptable de l'entreprise. À cet égard, nous attendons à ce que la qualité de l'information comptable soit positivement liée à la taille de l'entité qu'elle a produite (Chapellier et Mohammed, 2010).

\subsection{L'effet du degré de l'informatisation du service comptable d'une entreprise sur sa qualité de l'information comptable}

De toute évidence, la digitalisation des services de l'entreprise commence à prendre de l'ampleur. Le nombre des études qui s'intéressent aujourd'hui à l'impact de l'informatisation sur l'optimisation des services comptables est révélateur de l'important intérêt qu'offre l'outil informatique en termes d'accessibilité et d'efficacité des procédures.

C'est ainsi que les entreprises, même celles de petite taille, sont en situation de préparer leurs états de synthèses moyennant des progiciels comptables installés sur des machines informatiques. En fait, il faut souligner que l'informatisation de l'information comptable requière une maitrise de l'outil informatique. Pourtant l'informatisation du service comptable est tributaire de plusieurs avantages (Gingras et al., 1989) :

a. Elle offre la possibilité d'accéder simultanément à l'information par plusieurs utilisateurs ;

b. Elle facilite le contrôle des opérations et ;

c. Approuve la fiabilité et la véracité de l'information.

Pour toutes ces raisons, il est constaté que les entreprises ayant doter leurs services comptables de l'outil information, ont dû sacrifier l'ancienne structure organisationnelle du service pour y en bénéficier d'une réhabilitation marquante de la qualité de l'information préparer. Par conséquent, bénéficier d'une souplesse concernant le processus de prise des décisions justifiées en grande partie par des indicateurs à caractère comptable (Davis et Albright, 2000).

D'autant plus, l'outil informatique qui a fait ces débuts dans les organisations les plus structurées a fait preuve de son utilitarisme quant au raccourcissement des délais de traitement des volumes importants d'informations. De ce fait on a assisté à une consolidation des conditions de la prise des décisions de dirigeants (Orger, 1986). 
Toutefois, Dupuy (1983) souligne que la qualité de l'information comptable n'est pas constamment favorisée suite à l'intégration de l'outil informatique à la gestion du service comptable. Selon le même auteur, l'informatisation de l'information comptable se limite à la complaisance de l'usage de l'information, alors que la qualité de cette dernière est principalement la responsabilité des dirigeants. Dans notre travail, nous avons estimé bénéfique de mesurer l'impact, éventuel, que peut occasionner la présence d'un traitement informatisé de l'information au sein du service comptable. Pour ce faire nous avons intégré la variable « degré d'informatisation » dans le modèle d'évaluation des déterminants de la qualité de l'information comptable associée à la variable de l'intérêt principale. Étant donné le caractère latent de ladite variable, il a été nécessaire qu'on lui associer un ensemble d'indicateurs, issu de notre revue de littérature, en vue de rendre possible son appréciation sur le terrain à travers des traitements statistiques.

\subsection{L'effet de du profil du comptable sur la qualité de l'information comptable}

L'analyse de la qualité de l'information comptable s'est élargie au-delà du cadre de la théorie de la contingence structurelle, pour tenir en compte l'influence possible des acteurs intervenant à la production de l'information comptable (Chapellier, 1996 ; Bergeron, 1996 ; Lavigne, 2002 ; Flacke et Segbers, 2005 ; Nyengue Edimo, 2006 ; Affès et Chabchoub, 2007 ; Santin et Van Caillie, 2008).

En particulier, il s'agit d'introduire les traits du dirigeant et du comptable dans l'explication des disparités des résultats obtenus lors de l'étude de la qualité de l'information comptable de deux services comptable issus d'une population d'entreprises similaires. En somme c'est la théorie de la contingence comportementale qui vient remédier les insuffisances de la théorie de la contingence structurelle.

D'une manière ou d'une autre, la qualité de l'information comptable d'une entreprise est à la merci de la personne, généralement le comptable de l'entreprise, qui la prépare et la divulgue (Martel et al., 1985). Par ailleurs, le profil du comptable est réputé une clef de voute déterminante de la qualité de l'information. À partir de là, plusieurs auteurs ont exploré les différentes pistes envisageables en vue de fonder un bloc opératoire de manière à rendre commode la mesure de l'impact du profil du comptable sur la qualité de l'information comptable d'une entreprise (Nadeau et al., 1988 ; Julien et Marchesnay, 1988 ; Holmes et Nicholls, 1989 ; Holmes et al., 1991 ; Chapellier, 1994 ; Lavigne, 1999...).

La littérature se rapportant à cette section d'analyse mise en avant une batterie d'indicateurs qui s'attachent à rendre mesurable la variable « profil du comptable ». Dans ce sens, nous nous sommes inspirés des travaux de Chapellier (2013) pour soumettre ladite variable à l'évaluation.

\section{Construction du modèle hypothétique de la recherche}

Les études antérieures ont tenté ainsi d'identifier les déterminants de la qualité d'un système d'information comptable relatif aux facteurs de contingence structurelle, notamment la taille de l'entreprise, la forme juridique, l'organisation du service comptable, etc. (Michailesco 1998 ; 
Michailesco, 2000 Lassoued et Abdelmoula, 2006 ; Mahmoud, 2012 ; Hamadi, 2013 ; Super et Shil, 2017 ; Dieng, 2018). C'est ainsi que nous avons conçu la dimension « Organisation du service comptable de l'entreprise »(ORG_SER_COM_ETP) à travers laquelle nous envisageons évaluer l'impact des caractéristiques du service comptable sur le comportement de ses dirigeants quant à la production et la diffusion d'une information comptable de qualité supérieure. Étant donnée la nature endogène de la variable dépendante «Qualité de l'information comptable » que nous souhaitons expliquer, nous avons préludé son explication par une déclinaison en construit de deuxième ordre « Organisation du service comptable de l'entreprise » associer à trois variables de premier ordre : « Morphologie du service » (MOR_SER), « Degrés d'informatisation » (DEG_INF) et « Profil du comptable » (PRO_COM). La figure 23 illustre notre propos.

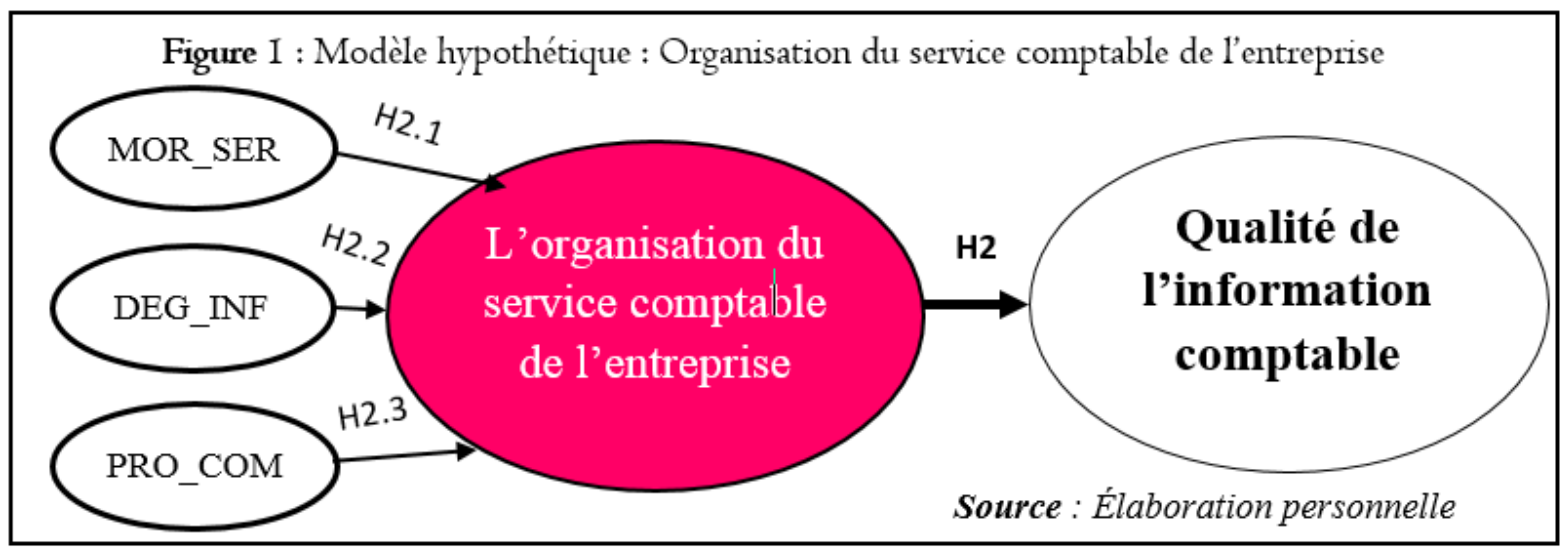

En somme, nous cherchons à travers l'élaboration du modèle à vérifier l'hypothèse suivante :

H 1 : l'organisation du service comptable de l'entreprise aurait un impact positif et significatif sur la qualité de l'information comptable.

\subsection{La morphologie du service comptable (MOR_SER)}

À travers la variable morphologie de service, nous tentons d'évaluer l'impact que peut avoir l'organisation de l'entité chargée de la production comptable sur la qualité de l'information comptable. Comme nous l'avons déjà mentionné, l'exploitation de ladite variable se heurte à la complexité de sa mesure, et par conséquent à son traitement statistique.

C'est ainsi qu'on doit passer par la déclinaison de la variable en indicateurs statistiques pertinents que nous avons pu repérer auparavant dans la revue de la littérature (Nyengue Edimo, 2006). Le tableau ciaprès relate les indicateurs dont nous nous sommes servies dans la collecte de données. 
Tableau I : Opérationnalisation de la variable " Morphologie du service "

\begin{tabular}{|c|c|c|}
\hline Dimension & Variable & Indicateurs \\
\hline $\begin{array}{l}\text { - Organisation du service } \\
\text { comptable }\end{array}$ & - Morphologie du service & 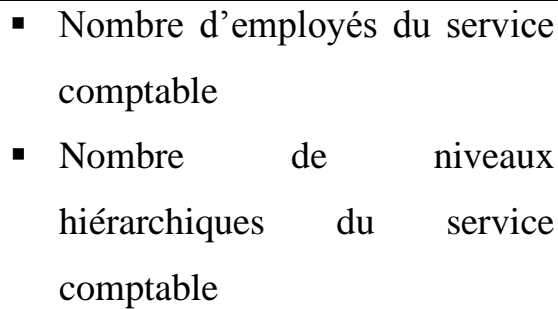 \\
\hline
\end{tabular}

Source : Élaboration personnelle

Nous retenons donc, les deux indicateurs (Nombre d'employés du service comptable et Nombre de niveaux hiérarchiques du service comptable) comme bloc de mesure de la variable (Morphologie du service). L'agglomération des deux indicateurs renseigne implicitement sur la taille du service comptable de l'entreprise. À cet égard, nous attendons à ce que la qualité de l'information comptable soit positivement liée à la taille de l'entité qu'elle a produite (Chapellier et Mohammed, 2010).

Ainsi on se propose donc de tester l'hypothèse suivante :

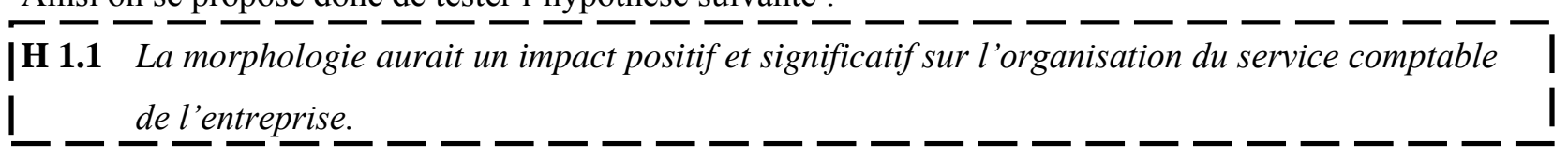

\subsection{Le degré de l'informatisation du service comptable (DEG_INF)}

De toute évidence, la digitalisation des services de l'entreprise commence à prendre de l'ampleur. Le nombre des études qui s'intéressent aujourd'hui à l'impact de l'informatisation sur l'optimisation des services comptables est révélateur de l'important intérêt qu'offre l'outil informatique en termes d'accessibilité et d'efficacité des procédures (Azhari et al., 2021).

C'est ainsi que les entreprises, même celles de petite taille, sont en situation de préparer leurs états de synthèses moyennant des progiciels comptables installés sur des machines informatiques. En fait, il faut souligner que l'informatisation de l'information comptable requière une maitrise de l'outil informatique. Pourtant l'informatisation du service comptable est tributaire de plusieurs avantages (Gingras et al., 1989) :
a. Elle offre la possibilité d'accéder simultanément à l'information par plusieurs utilisateurs ;
b. Elle facilite le contrôle des opérations et ;
c. Approuve la fiabilité et la véracité de l'information.

Pour toutes ces raisons, il est constaté que les entreprises ayant doter leurs services comptables de l'outil informatique, ont dû sacrifier l'ancienne structure organisationnelle du service pour y en bénéficier d'une réhabilitation marquante de la qualité de l'information préparer. Par conséquent, bénéficier d'une souplesse concernant le processus de prise des décisions justifiées en grande partie par des indicateurs à caractère comptable (Davis et Albright, 2000). 
D'autant plus, l'outil informatique qui a fait ces débuts dans les organisations les plus structurées a fait preuve de son utilitarisme quant au raccourcissement des délais de traitement des volumes importants d'informations. De ce fait on a assisté à une consolidation des conditions de la prise des décisions de dirigeants (Orger, 1986).

Toutefois, Dupuy (1983) souligne que la qualité de l'information comptable n'est pas constamment favorisée suite à l'intégration de l'outil informatique à la gestion du service comptable. Selon le même auteur, l'informatisation de l'information comptable se limite à la complaisance de l'usage de l'information, alors que la qualité de cette dernière est principalement la responsabilité des dirigeants.

Dans notre travail, nous avons estimé bénéfique de mesurer l'impact, éventuel, que peut occasionner la présence d'un traitement informatisé de l'information au sein du service comptable. Pour ce faire nous avons intégré la variable exogène de premier ordre « degré d'informatisation » dans le modèle associé à la dimension « Organisation du service comptable de l'entreprise ». Étant donné le caractère latent de ladite variable, il a été nécessaire qu'on lui associer un ensemble d'indicateurs (variables manifestes), issu de notre revue de littérature, en vue de rendre possible son appréciation sur le terrain à travers des traitements statistiques (Azhari, 2021).

Le tableau ci-après récapitule les indicateurs que nous avons retenus pour alléger l'assombrissement de la variable lors de la collecte des données (Chapellier et al., 2013).

Tableau 2 : Opérationnalisation de la variable " Degré d'informatisation "

\begin{tabular}{|l|l|l|}
\hline \multicolumn{1}{|c|}{ Dimension } & \multicolumn{1}{c|}{ Variable } & \multicolumn{1}{c|}{ Indicateurs } \\
\hline $\begin{array}{l}\text { - Organisation du service } \\
\text { comptable }\end{array}$ & - Degré d'informatisation & $\begin{array}{l}\text { - Nombre d'ordinateurs utilisés } \\
\text { - Usage de logiciel de } \\
\text { comptabilité }\end{array}$ \\
\hline
\end{tabular}

Source : Élaboration personnelle

Ainsi on se propose donc de tester l'hypothèse suivante :

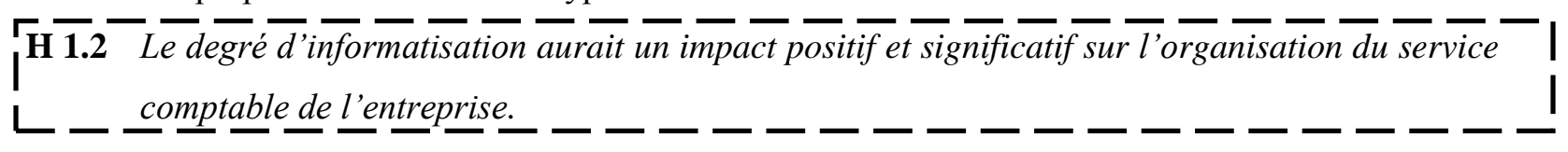

\subsection{Le profil du comptable (PRO_COM)}

L'analyse de la qualité de l'information comptable s'est élargie au-delà du cadre de la théorie de la contingence structurelle, pour tenir en compte l'influence possible des acteurs intervenant à la production de l'information comptable (Chapellier, 1996 ; Bergeron, 1996 ; Lavigne, 2002 ; Flacke et Segbers, 2005 ; Nyengue Edimo, 2006 ; Affès et Chabchoub, 2007 ; Santin et Van Caillie, 2008).

En particulier, il s'agit d'introduire les traits du dirigeant et du comptable dans l'explication des disparités des résultats obtenus lors de l'étude de la qualité de l'information comptable de deux services 
comptable issus d'une population d'entreprises similaires. En somme c'est la théorie de la contingence comportementale qui vient remédier des insuffisances de la théorie de la contingence structurelle.

D'une manière ou d'une autre, la qualité de l'information comptable d'une entreprise est à la merci de la personne, généralement le comptable de l'entreprise, qui la prépare et la divulgue (Martel et al., 1985). Par ailleurs, le profil du comptable est réputé une clef de voute déterminante de la qualité de l'information. À partir de là, plusieurs auteurs ont exploré les différentes pistes envisageables en vue de fonder un bloc opératoire de manière à rendre commode la mesure de l'impact du profil du comptable sur la qualité de l'information comptable d'une entreprise (Nadeau et al., 1988 ; Julien et Marchesnay, 1988 ; Holmes et Nicholls, 1989 ; Holmes et al., 1991 ; Chapellier, 1994 ; Lavigne, 1999...).

La littérature se rapportant à cette section d'analyse mise en avant une batterie d'indicateurs qui s'attachent à rendre mesurable la variable « profil du comptable ». Dans ce sens, nous nous sommes inspirés des travaux de Chapellier (2013) pour soumettre ladite variable à l'évaluation.

Le tableau ci-après reprend les indicateurs que nous avons retenus pour apaiser le haut niveau d'abstraction de la variable de sorte que l'on puisse la traiter d'une manière aisée sur le terrain.

Tableau 3 : Opérationnalisation de la variable "Profil du comptable "

\begin{tabular}{|c|c|c|}
\hline Dimension & Variable & Indicateurs \\
\hline $\begin{array}{l}\text { - Organisation du service } \\
\text { comptable }\end{array}$ & - Profil du comptable & $\begin{array}{l}\text { - Âge } \\
\text { - Expérience } \\
\text { - Niveau d'étude } \\
\text { - Domaine d'étude }\end{array}$ \\
\hline
\end{tabular}

Source : Élaboration personnelle

Ainsi on se propose donc de tester l'hypothèse suivante :

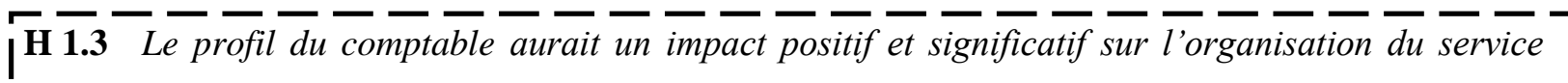
L - comptable de l'entreprise.

\section{Aspects épistémologiques et méthodologiques de l'étude}

\subsection{Posture épistémologique : positivisme, constructivisme ou paradigmes aménagés ?}

Rappelons ici que la perception de la réalité et la production de la connaissance y afférente s'inscrivent généralement, en sciences économiques, en deux perspectives épistémologiques principales : le positivisme et le constructivisme.

En ce qui nous concerne, la question que nous traitons et surtout son opacité nous impose de positionner notre recherche dans un paradigme post-positiviste. Il s'agit d'une version modérée du positivisme qui perçoit la réalité comme une vérité existante, mais qui accepte, quand même, l'incomplétude et la falsification dans la validation de la connaissance produite (Aissa, 2001). 


\subsection{Le positionnement méthodologique}

En sus du motif épistémologique susmentionné, nous aspirons à explorer la relation causale qui lie, d'un côté, la qualité de l'information comptable comme variable dépendante, et d'un autre côté, l'organisation du service comptable de l'entreprise comme variable explicative. Cela débute par un examen théorique menant à l'émission des hypothèses et finit par des tests empiriques réfutant ou corroborant les hypothèses émises. Par conséquence, nous nous inscrivons dans une démarche hypothético-déductive.

\subsection{La démarche méthodologique : quantitative ou qualitative ?}

Nos choix méthodologiques ont été largement influencés par les caractéristiques du matériau théorique à partir duquel nous avons construit notre modèle conceptuel de recherche. Notre cadre théorique est à la fois nourri par une analyse de la littérature et de l'observation de la discipline au cours de nos rencontres avec des experts comptables. La présente recherche portera ainsi sur des données numériques. Des données qui présentent deux avantages: D'un côté, elles sont susceptibles de quantifier nos concepts-clés, et d'un autre côté, elles sont appropriées aux analyses statistiques que nous souhaitons mener un peu plus loin. L'étude s'inscrit, par conséquent, dans une démarche quantitative.

\section{4.Échelle spatiale et procédure d'échantillonnage}

Notre échantillon est issu d'une base de données que nous avons pu constituer suite à nos visites aux quelques organismes spécialisés, notamment le tribunal du commerce d'Agadir, le centre régional d'investissement de la région Souss-Massa, la direction régionale du haut-commissariat au plan, la chambre de commerce, d'industrie et de services de Souss-Massa, la direction générale des impôts à Agadir et des bases de données des études antérieures. Pour identifier les entreprises agricoles, nous nous sommes inspirés de la loi n 112.12 du 18 Décembre 2014 promulguée par le Dahir ${ }^{\circ} 1$ - 83 - 226 du 9 moharram 1405 (5 octobre 1984) qui régit les coopératives. Cependant, il s'est avéré que le recensement des entreprises agricoles diffère d'un organisme à l'autre. Chose qui a débouché sur une population indéfinie, dont la taille réelle et méconnue. Face à une telle situation, la détermination d'un échantillon probabiliste a été inconcevable, en conséquence nous nous sommes retournés vers un type d'échantillonnage non aléatoire combinant les techniques d'échantillonnage par convenance et à boule de neige (Carricano et al., 2010). Sur la base de 321 questionnaires administrés, nous avons pu collecter un total de 226 questionnaires, soit un taux de retour de $70.4 \%$. Malgré le nombre des items constituant le questionnaire, $94,24 \%$ des répondants $(\mathbf{n}=\mathbf{2 1 3})$ l'ont renseigné en totalité.

\section{Analyse des données et discussion des résultats}

\section{1. Épuration des instruments des mesures}

À terme de notre choix de l'approche PLS, les lignes qui suivent s'attacheront à l'épuration des blocs de mesure associer à chacune des variables latentes du modèle de recherche. 


\subsection{1. Épuration des variables manifestes appréciant la variable latente «Morphologie du service »} Les items que nous avons mis en avant pour appréhender la variable latente «Morphologie du service » se présentent ainsi: NBR-EMP (Nombre d'employés) et NBR-NIV-HIE (Nombre de niveaux hiérarchique). La fiabilité et la validité interne de l'échelle de mesure que nous avons composée sont bien approuvées par un alpha de Cronbach de $\mathbf{0 . 8 7 7}$ (tableau 4).

Tableau 4 : Alpha de Cronbach calculé pour le bloc de mesure de la variable" Morphologie du service"

\begin{tabular}{|c|c|c|}
\hline \multicolumn{3}{|c|}{ Fiabilité et validité du construit } \\
\hline Construit & Alpha de Cronbach & Nombre d'éléments \\
\hline MOR-SER & $\mathbf{0 , 8 7 7}$ & 2 \\
\hline
\end{tabular}

Source : Résultats générés par SmartPLS

L'unidimensionnalité du bloc de mesure est acquittée par l'analyse en composantes principales qui restitue qu'une seule composante explique $\mathbf{8 9 \%}$ de la variance extraite du carré des changements (tableau 5).

Tableau 5 : Qualités des composantes de la variable latente « Morphologie du service » extraite par la méthode de l'ACP

\begin{tabular}{|c|c|c|c|c|c|c|}
\hline \multicolumn{7}{|c|}{ Variance totale expliquée } \\
\hline \multirow{3}{*}{ Composante } & \multicolumn{3}{|c|}{ Valeurs propres initiales } & \multicolumn{2}{c|}{ Sommes extraites du carré des chargements } \\
\cline { 2 - 7 } & Total & $\begin{array}{c}\text { \% de la } \\
\text { variance }\end{array}$ & $\%$ cumulé & Total & $\begin{array}{c}\text { \% de la } \\
\text { variance }\end{array}$ & $\%$ cumulé \\
\hline 1 & 1,781 & 89,025 & 89,025 & 1,781 & $\mathbf{8 9 , 0 2 5}$ & 89,025 \\
\hline 2 &, 219 & 10,975 & 100,000 & & & \\
\hline
\end{tabular}

\begin{tabular}{|c|c|c|}
\hline \multicolumn{3}{|c|}{ Qualités de représentation } \\
\hline & Initiales & Extraction \\
\hline NBR_EMP & 1,000 & $\mathbf{9 4 4}$ \\
\hline NBR_NIV_HIE & 1,000 & $\mathbf{9 4 4}$ \\
\hline
\end{tabular}

Source : Résultats générés par SPSS

En se basant sur les résultats obtenus de ces deux tests, la variable latente «Morphologie du service » sera par suite mesurée par les mêmes items proposés auparavant : NBR-EMP (Nombre d'employés) et NBR-NIV-HIE (Nombre de niveaux hiérarchique).

\subsection{2. Épuration des variables manifestes appréciant la variable latente «Degrés d'informatisation"}

«Degrés d'informatisation » est la deuxième variable latente sélectionnée pour apprécier la dimension «Organisation du service comptable de l'entreprise. ». Nous lui avons associé, antérieurement, deux items pour l'évaluer : NBR-ORD (Nombre d'ordinateurs) et LOG-COM (Usage de logiciel de 
comptabilité). La forte cohérence interne de l'instrument de mesure est approuvée par un alpha de Cronbach de 0,877 (Tableau 6).

Tableau 6 : Alpha de Cronbach calculé pour le bloc de mesure de la variable latente " Degrés d'informatisation "

\begin{tabular}{|c|c|c|}
\hline \multicolumn{3}{|c|}{ Fiabilité et validité du construit } \\
\hline Construit & Alpha de Cronbach & Nombre d'éléments \\
\hline DEG_INF & $\mathbf{0 , 8 7 7}$ & 2 \\
\hline
\end{tabular}

Source : Résultats générés par SmartPLS

Nous reprenons le même test de l'ACP au présent bloc de mesure afin de soutenir l'unidimensionnalité de l'instrument de mesure. Le test affirme qu'une seule composante explique plus de $\mathbf{8 9} \%$ de la variance (Tableau 7).

Tableau 7 : Qualités des composantes de la variable latente « Degrés d'informatisation » extraite par la méthode de l'ACP

\begin{tabular}{|c|c|c|c|c|c|c|}
\hline \multicolumn{7}{|c|}{ Variance totale expliquée } \\
\hline \multirow{3}{*}{ Composante } & \multicolumn{3}{|c|}{ Valeurs propres initiales } & \multicolumn{2}{c|}{ Sommes extraites du carré des chargements } \\
\cline { 2 - 7 } & Total & $\begin{array}{c}\text { \% de la } \\
\text { variance }\end{array}$ & \% cumulé & Total & $\begin{array}{c}\text { \% de la } \\
\text { variance }\end{array}$ & \% cumulé \\
\hline 1 & 1,781 & 89,053 & 89,053 & 1,781 & $\mathbf{8 9 , 0 5 3}$ & 89,053 \\
\hline 2 &, 219 & 10,947 & 100,000 & & & \\
\hline
\end{tabular}

\begin{tabular}{|c|c|c|}
\hline \multicolumn{3}{|c|}{ Qualités de représentation } \\
\hline & Initiales & Extraction \\
\hline NBR_ORD & 1,000 & $\mathbf{9 4 4}$ \\
\hline LOG_COM & 1,000 & $\mathbf{9 4 4}$ \\
\hline
\end{tabular}

Source : Résultats générés par SPSS

Le soubassement théorique solide de l'instrument de mesure de la variable latente "Degrés d'informatisation » est soutenu par les valeurs obtenues de son test empirique. Dès lors, nous maintenons les deux items NBR-ORD (Nombre d'ordinateurs) et LOG-COM (Usage de logiciel de comptabilité) comme bloc de mesure de la variable.

\subsection{3. Épuration des variables manifestes appréciant la variable latente «Profil du comptable »}

Le troisième construit décliné de la dimension « Organisation du service comptable de l'entreprise. » sollicite l'analyse de l'impact de la contingence comportementale que nous avons parcourue ci-devant au moyen de quatre variables manifestes : AGE (Age), EXP (Expérience), NIV-ETU (Niveau d'étude) et DOM-ETU (Domaine d'étude). La valeur de l'alpha de Cronbach (0.890) accrédite l'homogénéité des items sélectionnés, aussitôt, pour l'évaluation de la variable latente « Profil du comptable » (Tableau 8). 
Tableau 8 : Alpha de Cronbach calculé pour le bloc de mesure de la variable latente " Profil du comptable "

\begin{tabular}{|c|c|c|}
\hline \multicolumn{3}{|c|}{ Fiabilité et validité du construit } \\
\hline Construit & Alpha de Cronbach & Nombre d'éléments \\
\hline PRO-COM & $\mathbf{0 , 8 9 0}$ & 4 \\
\hline \multicolumn{3}{|c|}{ Source : Résultats générés par SmartPLS } \\
\hline
\end{tabular}

Le résultat obtenu du calcul de l'alpha de Cronbach est soutenu par l'ACP réalisé aux items. Ce dernier prouve qu'une seule composante explique plus de $\mathbf{7 5} \%$ de la variance avec des qualités de représentation soutenue (Tableau 9).

Tableau 9 : Qualités des composantes de la variable latente «Profil du comptable » extraite par la méthode de l'ACP

\begin{tabular}{|c|c|c|c|c|c|c|}
\hline \multirow{2}{*}{ Composante } & \multicolumn{5}{|c|}{ Valeurs propres initiance } & \multicolumn{2}{|c|}{ Sommes extraites du carré des chargements } \\
\cline { 2 - 6 } & Total & $\begin{array}{c}\text { \% de la } \\
\text { variance }\end{array}$ & \% cumulé & Total & $\begin{array}{c}\text { \% de la } \\
\text { variance }\end{array}$ & \% cumulé \\
\hline 1 & 3,008 & 75,194 & 75,194 & 3,008 & $\mathbf{7 5 , 1 9 4}$ & 75,194 \\
\hline 2 &, 443 & 11,065 & 86,259 & & \\
\hline 3 &, 342 & 8,558 & 94,817 & & \\
\hline 4 &, 207 & 5,183 & 100,000 & & \\
\hline
\end{tabular}

\begin{tabular}{|c|c|c|}
\hline \multicolumn{3}{|c|}{ Qualités de représentation } \\
\hline & Initiales & Extraction \\
\hline AGE & 1,000 & $\mathbf{8 9 8}$ \\
\hline EXP & 1,000 & $\mathbf{8 6 9}$ \\
\hline NIV_ETU & 1,000 & $\mathbf{8 6 6}$ \\
\hline DOM_ETU & 1,000 & $\mathbf{8 3 5}$ \\
\hline
\end{tabular}

Source : Résultats générés par SPSS

Subséquemment, l'analyse empirique de la variable latente « Structure de propriété » continuera avec les items déjà proposés dans la théorie : AGE (Age), EXP (Expérience), NIV-ETU (Niveau d'étude) et DOM-ETU (Domaine d'étude).

\section{L'estimation du modèle selon l'approche PLS : Un processus en deux étapes}

Les modèles de cheminement estimer sous l'approche PLS-PM sont constitués en deux éléments : (1) le modèle structurel qui décrit les relations entre les variables latentes, et (2) les modèles de mesure, qui décrivent les relations entre les variables latentes et leurs variables manifestes (c'est-à-dire leurs indicateurs). 


\subsection{Validation du modèle de mesure (Outer model)}

\subsubsection{Tests de fiabilité du modèle}

\subsubsection{Fiabilité composite des variables latentes d'ordre inférieur (Composite reliability)}

Les résultats calculés à partir de SmartPls témoignent une meilleure fiabilité composite pour notre cas d'analyse, c'est ainsi que tous les construits présentent une fiabilité de composite au-delà de 0.7 sans pour autant qu'elles dépassent le seuil non souhaitable de 0.95 .

Tableau I0 : Fiabilité composite des construits (CR)

\begin{tabular}{|c|c|c|}
\hline \multirow{2}{*}{ Variables latentes d'ordre inférieur } & \multicolumn{2}{|c|}{ Fiabilité des construits } \\
\hline & Alpha de Cronbach & Fiabilité Composite* \\
\hline MOR_SER & 0.877 & 0.942 \\
\hline DEG_INF & 0.877 & 0.942 \\
\hline PRO_COM & 0.890 & 0.924 \\
\hline
\end{tabular}

Source : Résultats obtenus à partir des calculs sous SmartPls

\subsubsection{Fiabilité des indicateurs (Loadings)}

La fiabilité des indicateurs réflexifs peut être appréciée à partir des poids de contribution factorielle (loadings) de chaque item. Attendu que les données sous SmartPls sont automatiquement normalisées, les charges externes (loadings) varient entre 0 et 1 . En principe, la fiabilité du modèle de mesure et d'autant solide au fur et à mesure que les loadings sont importants. Selon Hair et al., (2014), un item est en situation d'expliqué une grande partie de la variance du construit qui lui associer si la validité externe de ses charges est supérieure à 0,70 .

Les charges externes (loadings) doivent être en plus de valides $(\lambda \mathrm{i}>0.7)$ significatives. SmartPls offre au chercheur la possibilité d'évaluer l'importance et la pertinence du niveau de signification de chaque poids indicateur. En répliquant la taille de l'échantillon (5 000 fois ou plus), la technique de bootstrap permet d'obtenir un grand nombre de sous-échantillons à partir des données d'origine (avec remplacement) et des modèles d'estimation pour chaque sous-échantillon. Suit à cette opération, la signification de chaque paramètre du modèle (en particulier Outer loadings) est calculée sur la base des statistiques d'inférence afin de déterminer la contribution de chaque item à l'explication de construit lui associer (Hair et al., 2014). 
Tableau II : Fiabilité et signification des blocs de mesure (VM): Outer loadings et T Statistics

\begin{tabular}{|c|l|c|c|}
\hline \multirow{2}{*}{$\begin{array}{c}\text { Construits d'ordre } \\
\text { inférieur }\end{array}$} & \multicolumn{1}{c|}{ Items } & \multicolumn{2}{c|}{ Fiabilité des indicateurs } \\
\cline { 2 - 4 } & \multicolumn{1}{c|}{$\begin{array}{c}\text { Toadings }(\lambda \mathbf{i}) \\
\mathbf{a}\end{array}$} & $\begin{array}{c}\text { T Statistics } \\
\text { (Signification) }\end{array}$ \\
\hline \multirow{2}{*}{ MOR-SER } & NBR_EMP <- MOR-SER & 0.942 & $02.524 * *$ \\
\cline { 2 - 4 } & NBR_NIV_HIE <- MOR-SER & 0.945 & $02.607 * * *$ \\
\hline \multirow{2}{*}{ DEG-INF } & NBR_ORD <- DEG-INF & 0.955 & $06.288 * * *$ \\
\cline { 2 - 4 } & LOG_COM <- DEG-INF & 0.932 & $05.391 * * *$ \\
\hline \multirow{2}{*}{ PRO-COM } & AGE <- PRO-COM & 0.896 & $10.478 * * *$ \\
\cline { 2 - 4 } & EXP <- PRO-COM & 0.870 & $10.457 * * *$ \\
\cline { 2 - 4 } & NIV_ETU <- PRO-COM & 0.868 & $10.353 * * *$ \\
\cline { 2 - 4 } & DOM_ETU <- PRO-COM 0.834 & $10.089 * * *$ \\
\hline
\end{tabular}

Source : Résultats obtenus à partir des calculs sous SmartPls

\subsubsection{Test de la validité convergente}

La validité convergente signifie que le bloc de mesure (indicateurs) appartenant à une variable latente mesure un seul et même construit. La variance moyenne extraite (AVE) est le critère par excellence utilisé pour l'évaluation de la validité convergente (Fornell et Larcker, 1981). Un AVE supérieur à $\mathbf{0 . 5}$ a été déterminé comme seuil suffisant à fournir des preuves empiriques de la validité convergente. Statistiquement, cela revient à dire que le construit en question est capable d'expliqué plus de la moitié de la variance de ses indicateurs en moyenne et, par conséquent, toutes les autres variables latentes expliquent moins de la moitié d'entre elles (Benitez et al., 2020). Pour notre cas, toutes les valeurs de l'AVE sont supérieures à $0, \mathbf{7 5 0}$, dès lors tous les construits de notre modèle de mesure expliquent plus de $\mathbf{7 5 \%}$ de leurs variables manifestes (Items).

Tableau I2: Test AVE de la validité convergente

\begin{tabular}{|c|c|}
\hline Construits d'ordre inférieur & Average Variance Extracted (AVE)* \\
\hline MOR-SER & 0.890 \\
\hline DEG-INF & 0.890 \\
\hline PRO-COM & 0.752 \\
\hline *Seuil recommandé $: A V E>0.5$ & \\
\hline
\end{tabular}

Source : Résultats obtenus à partir des calculs sous SmartPls 


\subsubsection{Test de la validité discriminante}

\subsubsection{Validité discriminante au niveau des construits (Test de Fornell et Larcker)}

Une première sortie que propose SmartPls pour vérifier la validité discriminant des construits d'un modèle est le test de Fornell et Larcker. Pratiquement ce test exige que pour tous construit (VL), la variance partagée avec son bloc de mesure (Items) doit être supérieure à la variance qu'il partage avec toute autre variable latente (Hair et al., 2014). Tel est bien la situation, l'application du test consolide la validité discriminante de notre modèle de mesure.

Tableau I3 : Test de Fornell et Larcker de validité discriminante

\begin{tabular}{|l|c|c|c|}
\hline & DEG-INF & MOR-SER & PRO-COM \\
\hline DEG-INF & $\mathbf{0 . 9 4 3}$ & & \\
MOR-SER & 0.146 & $\mathbf{0 . 9 4 4}$ & \\
PRO-COM & 0.146 & 0.200 & $\mathbf{0 . 8 6 7}$ \\
\hline
\end{tabular}

Source : Résultats obtenus à partir des calculs sous SmartPls

\subsubsection{Validité discriminante au niveau des indicateurs (Test de Cross-Loadings)}

Le test de cross-loadings offre une deuxième alternative pour vérifier la validité discriminante du modèle de mesure (Götz et al., 2009). Dans un bon modèle, les loadings (poids externes) d'un indicateur sont censés être plus élevés que ces poids croisés (cross-loadings) (Chin, 1998). Au gré des critères recommandés ci-devant, la validité discriminante de notre modèle de mesure est proche de l'idéal que décrit Garson (2016). Le tableau ci-après illustre ce propos.

Tableau I4 : Test de cross-loadings de validité discriminante

\begin{tabular}{l|c|c|c|}
\hline & MOR-SER & \multicolumn{1}{c}{ DEG-INF } & PRO-COM \\
\hline NBR_EMP & $\mathbf{0 . 9 4 2}$ & 0.126 & 0.182 \\
NBR_NIV & $\mathbf{0 . 9 4 5}$ & 0.148 & 0.196 \\
NBR_ORD & 0.164 & $\mathbf{0 . 9 5 5}$ & 0.162 \\
LOG_COM & 0.105 & $\mathbf{0 . 9 3 2}$ & 0.109 \\
AGE & 0.141 & 0.111 & $\mathbf{0 . 8 9 6}$ \\
EXP & 0.219 & 0.078 & $\mathbf{0 . 8 7 0}$ \\
NIV_ETU & 0.178 & 0.197 & $\mathbf{0 . 8 6 8}$ \\
DOM_ETU & 0.155 & 0.120 & $\mathbf{0 . 8 3 4}$
\end{tabular}

Source : Résultats obtenus à partir des calculs sous SmartPls 
Le modèle de mesure (modèle externe) étant validé, nous nous tournons maintenant à la validation du modèle structural (modèle interne). Mais avant de nous nous engageons dans la deuxième phase, nous tenons à passer en revue, de manière schématique, les différentes étapes que nous avons affranchies pour en arriver là. La figure ci-après relate brièvement nos propos.

Figure 2 : le «Path modeling » de notre modèle hiérarchique sous PLS en utilisant l'approche des indicateurs répétés

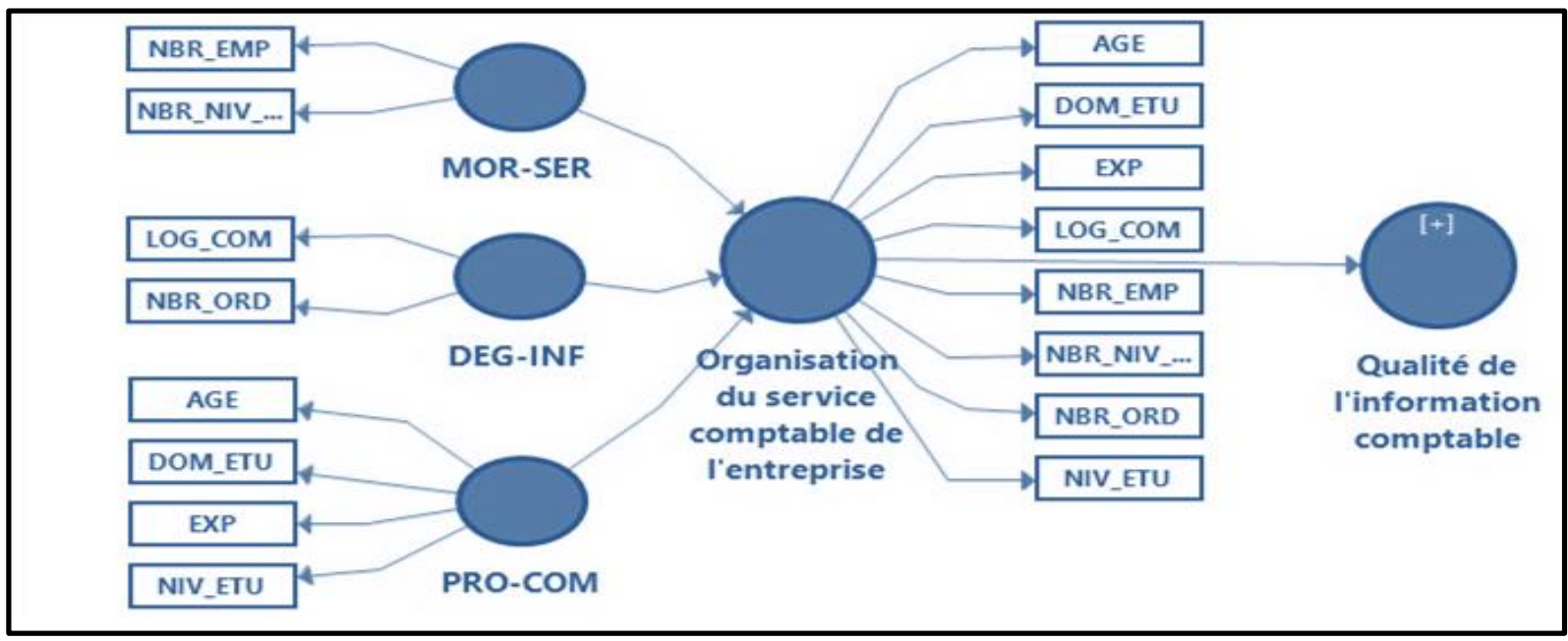

Source : Élaboration personnelle sous SmartPLS

\subsection{Validation du modèle structurel (Inner model)}

\subsubsection{Test de multicolinéarité : facteur d'inflation de la variance}

Nous commençons le processus de l'évaluation du notre modèle structurel par un contrôle du niveau de multicolinéarité que présente nos construits formatifs. L'indice communément utilisé pour la vérification de la multicolinéarité est le coefficient du facteur d'inflation de la variance (VIF). L'application du test du VIF sur notre modèle structurel a révélé des niveaux très bas de multicolinéarité largement au-dessous des seuils recommandés. Ceci est valable pour la dimension du deuxième ordre $($ ORG_SER_COM $<2)$, comme pour le construit du troisième ordre (Qualité de l'information comptable : QIC < 2).

Tableau I5 : Collinearity Statistics (Les valeurs du VIF du modèle interne)

\begin{tabular}{|c|c|c|}
\hline \multicolumn{3}{|c|}{ Collinearity Statistic - Inner VIF ${ }^{a}$ Values } \\
\hline \multicolumn{3}{|c|}{ Second-order construct } \\
\hline & ORG_SER_COM & QIC \\
\hline MOR-SER & 1.057 & \\
\hline DEG-INF & 1.037 & \\
\hline PRO-COM & 1.057 & \\
\hline \multicolumn{3}{|c|}{ Third-order construct } \\
\hline ORG_SER_COM & & 1.040 \\
\hline
\end{tabular}

Source : Résultats obtenus à partir des calculs sous SmartPls 


\subsubsection{Coefficients de chemin du modèle structurel et résultats des tests de signification}

\subsubsection{1 Évaluation des coefficients de chemin (Path Coefficients)}

D'un point de vue statistique, les coefficients de chemin sont généralement compris entre -1 et +1 , les coefficients plus proches de $\boldsymbol{+ 1}$ représentant des relations positives fortes, et ceux plus proches de $\mathbf{- 1}$ indiquant des relations négatives fortes ${ }^{1}$ (Sarstedt et al., 2017). Chin (1998) considère que «les coefficients structurels standardisés devraient être au minimum égal à $\mathbf{0 . 2}$, et, idéalement, supérieur à 0.3 pour pouvoir être considérés comme significatifs ». Touchant à notre modèle structurel, l'analyse des coefficients structurels témoigne des relations positives très significatives raccordant les variables latentes d'ordre inférieur à la dimension du second ordre. De même l'association de notre dimension du deuxième ordre à la qualité de l'information comptable révèle une relation très significative.

Tableau I6: Performance des coefficients de chemin du modèle structurel

\begin{tabular}{|l|c|c|}
\hline & ORG_SER_COM & QIC \\
\hline \multicolumn{3}{|c|}{ Second-Order Construct } \\
\hline MOR_SER & $0.433^{* * *}$ \\
\hline DEG_INF & $0.210^{* *}$ & $0.752^{* * *}$ \\
\hline PRO_COM & Third-Order Construct \\
\hline \multicolumn{3}{|c|}{ Effets directs } \\
\hline ORG_SER_COM & Effets indirects \\
\hline \multicolumn{2}{|c|}{$0.305^{* * *}$} \\
\hline MOR_SER & 0.132 \\
\hline DEG_INF & 0.064 \\
\hline PRO-COM & & $0.229^{* *}$ \\
\hline
\end{tabular}

Source : Résultats obtenus à partir des calculs sous SmartPls

\subsubsection{Test de signification des coefficients de chemin du modèle structurel}

Tenenhaus et al., (2005) recommandent de compléter l'analyse précédente par une évaluation des niveaux de significativité des coefficients de régression obtenus moyennant des techniques de réplication des échantillons, tels que le bootstrapping afin de déterminer les intervalles de confiance des coefficients de chemin et consolider la signification des liaisons entre les construit du modèle.

Tableau I7 : Test de signification des coefficients de chemin du modèle structurel

\begin{tabular}{|l|c|c|c|}
\hline Means, STDEV, T-Values, P-Values & a Path coefficients & ${ }^{\text {b}}$ T Statistics $(|\mathbf{O} / \mathbf{S T D E V}|)$ & P Values \\
\hline \multicolumn{5}{|c|}{ Second-Order Construct } \\
\hline MOR-SER -> ORG_SER_COM & $0.433 * * *$ & 2.531 & 0.011 \\
\hline DEG-INF -> ORG_SER_COM & $0.210 * * *$ & 2.687 & 0.007 \\
\hline PRO-COM -> ORG_SER_COM & $0.752 * * *$ & 6.937 & 0.000 \\
\hline
\end{tabular}

\footnotetext{
${ }^{1}$ (Notez que des valeurs inférieures à -1 et supérieures à +1 peuvent techniquement se produire, par exemple, lorsque la colinéarité est à des niveaux critiques)
} 


\begin{tabular}{|l|c|c|c|}
\hline \multicolumn{4}{|c|}{ Third-Order Construct } \\
\hline \multicolumn{4}{|c|}{ Effets directs } \\
\hline ORG_SER_COM-> QIC & 0.305 NS & 1.353 & 0.176 \\
\hline \multicolumn{3}{|c|}{ Effets indirects } \\
\hline DEG-INF -> QIC & 0.064 & 1.199 & 0.231 \\
\hline PRO-COM -> QIC & 0.229 & 1.267 & 0.205 \\
\hline MOR-SER -> QIC & 0.132 & 1.644 & 0.100 \\
\hline $\begin{array}{l}\text { a Significativité au seuil } * * * p<0.001 \\
\text { b }(t>2.57) ; * * p<0.01(t>1.96) ; * p<0.1 \quad(t>1.65)\end{array}$ \\
\hline
\end{tabular}

Source : Résultats obtenus à partir des calculs sous SmartPls

Lors de l'analyse des estimations des coefficients de régressions du modèle structurel, nous constatons que l'ensemble des variables latentes d'ordre inférieur contribuent significativement (au seuil de 1\%) à la dimension du second ordre (ORG_SER_COM). La variable latente PRO_COM (0.752) est celle qui contribue le plus à la construction de la dimension (ORG_SER_COM). Au niveau du troisième ordre, les résultats du bootstrapping (une réplication à $\mathrm{N}=5000$ ) confirment que la dimension du deuxième niveau ORG_SER_COM (0.305) a un effet positif, mais relativement non significatif au niveau de probabilité d'erreur de $1 \%(\mathrm{t}>2.58)$ sur la variable endogène d'ordre supérieur QIC.

Nous pouvons ainsi avancer, dans le cadre des effets indirects du modèle structurel, que les variables latentes d'ordre inférieur PRO-COM (0.229) forme pertinemment la qualité de l'information comptable (ordre supérieur).

\subsubsection{Test des hypothèses associées aux modèles réduits}

Le test de signification des coefficients de chemin du modèle structurel nous a permis de nous assurer de la plausibilité de notre modèle hypothétique globale. Nous nous tournons à présent au test des hypothèses à travers l'évaluation des Std Beta de chaque lien structurel formant les hypothèses de notre modèle. Nous interpellons ainsi une réplication de notre échantillon suivant la technique du bootstrap $(\mathrm{N}=5000)$ en vue d'apprécier la signification des liens surgissants.

La lecture du tableau ci-après nous indique que trois hypothèses du modèle sont supportées (2 hypothèses au seul d'erreur de $1 \%$ et 1 au seuil d'erreurs de $5 \%$ ) et une seule hypothèse non supportée. La discussion en détail de ces relations hypothétique fera l'objet d'un point sur lequel nous reviendrons au moment où nous discuterons nos résultats du modèle (Azhari, 2021).

Tableau 18 : Test des hypothèses associées au modèle global

\begin{tabular}{|c|l|c|c|c|c|c|}
\hline Hyp. & \multicolumn{1}{|c|}{ Liens structurels } & Std. Beta & $\begin{array}{c}\text { Std. } \\
\text { Error }\end{array}$ & T-Value & P-Value & Décision \\
\hline H 1.1 & MOR-SER $\rightarrow$ ORG_SER_COM & $\mathbf{0 . 4 3 3} * *$ & $\mathbf{0 . 1 7 3}$ & $\mathbf{2 . 5 0 6}$ & $\mathbf{0 . 0 1 2}$ & Acceptée $* *$ \\
\hline H 1.2 & DEG-INF $\rightarrow$ ORG_SER_COM & $\mathbf{0 . 2 1 0} * * *$ & $\mathbf{0 . 0 8 1}$ & $\mathbf{2 . 5 9 3}$ & $\mathbf{0 . 0 1 0}$ & Acceptée $* *$ \\
\hline H 1.3 & PRO-COM $\rightarrow$ ORG_SER_COM & $\mathbf{0 . 7 5 2} * * *$ & $\mathbf{0 . 1 1 3}$ & $\mathbf{6 . 6 7 2}$ & $\mathbf{0 . 0 0 0}$ & Acceptée $* * *$ \\
\hline H 1 & ORG_SER_COM $\rightarrow$ QIC & $\mathbf{0 . 3 0 5}$ NS & $\mathbf{0 . 2 2 7}$ & $\mathbf{1 . 3 4 2}$ & $\mathbf{0 . 1 8 0}$ & Rejetée \\
\hline
\end{tabular}




\subsubsection{Le coefficient de détermination ( $R$ square)}

Nous rappelons que nous avons conçu notre modèle hiérarchique à variable latente selon l'approche des indicateurs répétés suivant un modèle de type II (réflectif-formatif). Dans telles circonstances, la quasitotalité de la variance des construits d'ordre supérieur (HOC) est expliquée par ses construits sous-jacent d'ordre inférieur (LOC) générant, par conséquent, des valeurs de $\mathrm{R}^{2}$ proche de $1\left(\mathrm{R}^{2} \approx 1\right)$ (Ringle et al., 2012). Tel est bien le cas, le tableau ci-après illustre ce que nous avons prévu précédemment.

Tableau 19 : Test de coefficient de détermination ( $R$ square)

\begin{tabular}{|c|c|c|}
\hline \multicolumn{3}{|l|}{$R$ Square } \\
\hline & ${ }^{\text {a }}$ R Square & R Square Adjusted \\
\hline \multicolumn{3}{|c|}{ Second-Order construct } \\
\hline ORG_SER_COM & 1.000 & 1.000 \\
\hline \multicolumn{3}{|c|}{ Third-Order construct } \\
\hline QIC & 0.988 & 0.988 \\
\hline
\end{tabular}

Source : Résultats obtenus à partir des calculs sous SmartPls

\subsection{4. Évaluation de l'ampleur de l'effet $\left(f^{2}\right)$}

Touchant à notre modèle structurel, l'évaluation des coefficients de la détermination $\mathrm{R}^{2}$ a attesté que les construits d'ordre supérieur sont parfaitement expliqués par leurs sous-dimensions $\left(\mathrm{R}^{2} \approx 1.0\right)$, c'est la raison pour laquelle nous présumons à ce que l'ampleur des effets de nos construits hiérarchiques sera traduite par des valeurs de $f^{2}$ très importantes par rapport aux seuils recommandés. Une hypothèse que nous avons confirmée plus tard par les valeurs étalées sur le tableau ci-après.

Tableau 20 : Évaluation des effets de taille (f $\left.\mathbf{f}^{2}\right)$

\begin{tabular}{|c|c|c|}
\hline \multicolumn{3}{|l|}{$F$ square $\left(f^{2}\right)$} \\
\hline & ORG_SER_COM & QIC \\
\hline \multicolumn{3}{|c|}{ Second-Order Construct } \\
\hline MOR_SER & \multicolumn{2}{|l|}{12806.103} \\
\hline DEG_INF & \multicolumn{2}{|l|}{3082.751} \\
\hline PRO_COM & \multicolumn{2}{|l|}{38650.933} \\
\hline \multicolumn{3}{|c|}{ Third-Order Construct } \\
\hline ORG_SER_COM & & 7.242 \\
\hline Significativité $: f^{2}>$ & & \\
\hline
\end{tabular}

Source : Résultats obtenus à partir des calculs sous SmartPls

Les effets de taille calculés pour notre modèle structurel sont dans l'ensemble très importants. L'importance de ces effets s'explique par les niveaux élevés des coefficients de détermination $\mathrm{R}^{2}$ calculés ci-devant. Si nous nous intéressons précisément aux relations structurelles reliant la dimension ORG_SER_COM à ses différentes variables qui lui sont liées, nous pouvons dire que les trois variables latentes d'ordre inférieur MOR_SER (12806.103***), DEG_INF (3082.751***) et PRO_COM (38650.933***) ont tous un effet très étendu sur leur dimension (construit du deuxième ordre).

6.2.5. Test de la pertinence prédictive du modèle $Q^{2}$ : (predictive relevance) 
Il est d'usage apprécier à travers la mesure prédominante de la pertinence prédictive le $\mathrm{Q}^{2}$ de StoneGeisser (Stone, 1974 ; Geisser, 1975). La valeur Q² des variables latentes dans le modèle de chemin PLS est obtenue en utilisant la procédure du Blindfolding2. Plus la valeur est éloignée de 0, plus la fiabilité prédictive des estimations du construit est assurée. La procédure du Blindfolding ne peut être appliquée qu'aux variables endogènes latentes qui ont un modèle de mesure réflexif. Une condition que notre modèle remplit grâce à la technique des indicateurs répétés, même si nous adaptons un schéma formatif reliant les construits du deuxième et troisième ordre à leurs variables sous-jacent. Le tableau ci-après reprend les valeurs $\mathrm{Q}^{2}$ obtenues.

Tableau 21 : Test de la pertinence prédictive par validation croisée de Stone- Geisser $\mathbf{Q}^{2}$

\begin{tabular}{|l|c|c|c|}
\hline \multicolumn{2}{|c|}{ Construct Cross-validated redundancy } & SSE & ${ }^{{ }^{a}} \mathbf{Q}^{2}$ (=1-SSE/SSO) \\
\hline \multicolumn{4}{|c|}{ Second-Order construct } \\
\hline ORG_SER_COM & 1704.000 & 1078.783 & $0.367^{* * *}$ \\
\hline \multicolumn{2}{|c|}{ Third-Order construct } \\
\hline QIC & 4899.000 & 4158.228 & $0.151^{* *}$ \\
\hline${ }^{a}$ Seuil recommandé $: Q^{2}>0$ & \multicolumn{3}{|}{} \\
\hline
\end{tabular}

Les résultats obtenus du Blindfolding pour le test de $\mathrm{Q}^{2}$ de Stone-Geisser affichent des valeurs de $\mathrm{Q}^{2}$ qui dépassent largement 0 . C'est ainsi que la performance de la prédictibilité du nôtre modèle se trouve soutenue. Cette performance est aperçue au niveau des construits du second (ORG_SER_COM: $Q^{2}=$ $\left.0.367^{* * *}\right)$ comme pour la variable endogène du troisième ordre $\left(Q I C: Q^{2}=0.151^{* *}\right)$.

\section{Discussion des résultats}

Le présent paragraphe mène une discussion des différents éléments, que nous avons engagée précédemment, on les confrontant à la littérature antérieure. Pour ce faire, un retour sera fait sur les premiers résultats de notre analyse ayant intéressé la validité et la fiabilité des instruments de mesure. Nous poursuivons notre discussion par une mise en perspective des liens structurels directe (hypothèses) qui sont établies entre les construits des différents niveaux hiérarchiques de notre modèle. À juste titre, nous allons déclencher la discussion sur les résultats de chaque hypothèse, en prenant soin de les comparer aux travaux antérieurs.

\subsection{Effets de l'organisation du service comptable de l'entreprise sur la QIC}

L'étape préalable à l'analyse de l'impact de ces variables sur la dimension « ORG_SER_COM » nous a rassurés sur la fiabilité interne des indicateurs et l'unidimensionnalité des blocs de mesure. C'est ainsi que le recours à l'indice classique de mesure de la cohérence interne alpha de Cronbach, consolider par l'indice de la fiabilité composite ne serait-ce que pour légitimer notre démarche et garantir une teneur

\footnotetext{
${ }^{2}$ C'est une technique de réutilisation d'échantillons, qui supprime systématiquement les points de données et permet de prédire leurs valeurs initiales (Hair et al., 2017)
} 
scientifique à nos propos. De par les valeurs calculées (Alpha de Cronbach MOR_SER $=0.877$; Fiabilité Composite MOR_SER $=0.942)$, (Alpha de Cronbach DEG_INF $=0.877$; Fiabilité Composite DEG_INF $=0.942)$ et $($ Alpha de Cronbach PRO_COM $=0.890$; Fiabilité Composite PRO_COM = 0.924), nous contemplons, donc, que les construits d'ordre inférieur que nous avons proposé pour mesurer la dimension «ORG_SER_COM » possèdent suffisamment d'uniformité pour sauvegarder la teneur théorique du construit.

\subsubsection{Variables manifestes appréciant la dimension «ORG_SER_COM » : fiable et significative}

À l'effet du haut niveau d'abstraction que présentent les variables latentes d'ordre inférieur, il a été nécessaire de décliner ces derniers en variables manifestes (indicateurs) pour que l'on puisse les traiter empiriquement d'une manière aisée. Pour vérifier la disposition de ses indicateurs à mesurer ce qu'ils sont censés mesurer, nous les avons soumis à deux tests statistiques, respectivement, le poids de contribution factorielle (loadings) et test de signification moyennant la technique de bootstrap (à $\mathrm{N}=5000$ réplications). Les résultats obtenus corroborent la capacité de nos blocs de mesure à répondre aux exigences empiriques de l'analyse.

En particulier, les items liés à la variable (MOR_SER) ont enregistré les valeurs suivantes : (Loadings NBR_EMP <- MOR-SER $=0.942 ;$ t-value NBR_EMP <- MOR-SER $=02.524 * *)$ et (Loadings NBR_NIV <- MOR-SER $=0.945$; t-value NBR_NIV <- MOR-SER $=02.607 * *$ ). Pour leurs parts, les valeurs qu'affichent les items associés à la variable (DEG_INF) témoignent leurs profondes contributions à expliquer cette dernière : (Loadings NBR_ORD <- DEG-INF $=0.955 ; \mathrm{t}$-value NBR_ORD <- DEG-INF $=06.288 * * *)$ et (Loadings LOG_COM <- DEG-INF $=0.932 ; \mathrm{t}$-value

LOG_COM <- DEG-INF $=02.607 * *$ ). La dernière variable $«$ PRO_COM $»$ qui introduit les traits du dirigeant et du comptable dans l'explication de la qualité de l'information comptable a été déclinée, à son tour, à quatre variables manifestes dont le test de validité interne a surgi sur les résultats suivants : (Loadings AGE <- PRO-COM= 0.896 ; t-value AGE <- PRO-COM = $10.478 * * *$ ), (Loadings EXP <PRO-COM $=0.870 ; \mathrm{t}$-value EXP <- PRO-COM = $10.457 * * *$ ), (Loadings NIV_ETU <- PRO-COM = 0.868 ; t-value NIV_ETU <- PRO-COM $=10.353 * * *)$ et (Loadings DOM_ETU <- PRO-COM $=0.834$ ; t-value DOM_ETU <- PRO-COM = $10.089 * * *)$.

\subsubsection{La contribution des variables latentes d'ordre inférieur à l'explication de la dimension « ORG_SER_COM »}

Restant, toujours, au niveau des relations structurelles entre les construits de premier ordre et les construits de second ordre «ORG_SER_COM». Les trois variables latentes que nous avons choisies pour former la présente dimension sont ((MOR_SER), (DEG_INF) et (PRO_COM)). La première variable latente (MOR_SER) évalue l'impact que peut avoir l'organisation de l'entité chargée de la production comptable sur la qualité de l'information comptable. Ce premier lien structurel atteste que la variable latente (MOR_SER) contribue significativement et pertinemment (MOR_SER 
ORG_SER_COM : $\beta=0.433 ;$ Std. Error $\left.=0.173 ; \mathrm{t}=2.506^{* *} ; \mathrm{p}<0.05\right)$ à la composition de la dimension «ORG_SER_COM».

Nos résultats rejoignent, sur ce point, ceux des rares études antérieures (Nobre, 2001 ; Chenhall, 2003 ; Mintzberg, 2006 ; Abdel Kader et Luther, 2008). Ils expliquent que l'accroissement de la taille emmène, généralement, à l'évolution des outils comptables de gestion présente au service comptable ce qui entraine une amélioration de la qualité de l'information comptable préparé. Cet aboutissement est validé en partie par Djongoué (2015) qui, en se basant sur les résultats de (Ijiri et al., 1966 ; william et al., 1968 ; casey, 1978 ; Edmonds ,1981 ; Dyckman et al., 1982 ; Munter et al., 1983, Chung et al., 1984 ; Bouwman et al., 1987), soutient cette assertion théorique.

Le deuxième lien structurel rencontre les préoccupations théoriques qui avancent que les entreprises ayant doter leurs services comptables de l'outil information et sacrifier l'ancienne structure organisationnelle du service devraient bénéficier d'une réhabilitation significative de la qualité de l'information préparer. La contribution de la variable « DEG_INF », qui concrétise ladite relation, est suffisamment forte à la composition de la dimension «ORG_SER_COM». Les résultats obtenus illustrent ce propos (DEG-INF $\rightarrow$ ORG_SER_COM: $\beta=0.210$; Std. Error $=0.081 ; t=2.593 * * ; p<$ $0.05)$.

Ce constat obtenu, à ce niveau, rejoint ceux des différentes recherches qui soutiennent le rôle de l'outil informatique à la consolidation de la qualité de l'information comptable d'une entreprise. Parmi ces recherches, on trouve Gingras et al., (1989) qui affirment que la digitalisation de service comptable approuve la fiabilité et la véracité de l'information comptable produite. Orger (1986) et Davis et Albright (2000) vont dans le même sens et confirment que l'outil informatique structuré a fait preuve de son utilitarisme quant au raccourcissement des délais de traitement des volumes importants d'informations comptables. Pour sa part, Dupuy (1983) réfute catégoriquement l'accouplement de la qualité de l'information comptable au degré de l'informatisation du service comptable. Pour lui l'informatisation de l'information comptable se limite à la complaisance de l'usage de l'information, alors que la qualité de cette dernière est principalement la responsabilité des dirigeants.

Parmi les buts recherchés par ce modeste travail est de pouvoir évaluer les contributions de la théorie de la contingence comportementale pour se remédier aux insuffisances de la théorie de la contingence structurelle. En particulier, il s'agit d'introduire les traits du comptable dans l'explication lors de l'étude de la qualité de l'information comptable. C'est pour cette fin que nous avons composé une troisième variable latente d'ordre inférieur que nous avons intitulé le profil du comptable (PRO_COM).

L'analyse de coefficient de chemin montre que la variable (PRO_COM) contribue substantiellement et significativement à la formation de la dimension «ORG_SER_COM». Les résultats obtenus classent la variable (PRO_COM) en première position en termes de sa contribution à l'explication de la variance de la dimension «ORG_SER_COM» $($ PRO-COM $\rightarrow$ ORG_SER_COM $: \beta=0.752$; Std. Error $=0.113$ 
$; \mathrm{t}=6.672 * * * ; \mathrm{p}<0.001)$. Certaines études soutiennent nos propos, dont celle de Chapelier et al., (2013) qui établit une relation positive et significative entre les items (le niveau de formation du comptable, la mission du comptable et l'implication de l'expert-comptable) et la qualité de l'information comptable préparée. Les conclusions de (Nadeau et al., 1988 ; Julien et Marchesnay, 1988 ; Holmes et Nicholls, 1989 ; Holmes et al., 1991 ; Chapellier, 1994 ; Lavigne, 1999 et Martel et al., 1985) et ceux de notre étude semblent être également comparable.

De prime abord, et en regard de l'ampleur et la significativité de l'effet des liens structurels ayant été vérifié au niveau inférieur (H1.1 ; H1.2 et H1.3), nous attendions à ce que la dimension « ORG_SER_COM»contribue dans les mêmes proportions, que ces sous-dimensions, à la formation de la variable d'ordre supérieur (QIC). Par contre, le résultat de coefficient de chemin calculé pour la dimension «ORG_SER_COM» est surprenant, puisque cette hypothèse semble évidente. En réalité cette dernière maintient le même sens qu'au niveau inférieur, mais perdre de significativité quant à l'explication de la variance de la variable endogène QIC $($ ORG_SER_COM $\rightarrow$ QIC : $\beta=0.305$; Std. Error $=0.227 ; \mathrm{t}=1.342 ; \mathrm{p}>0.100)$.

La remise en cause de l'hypothèse $\mathrm{H} 1$ est d'autant plus étonnante, dans la mesure où elle s'oppose aux aboutissements développés par de Michailesco (1998) et de Mahmoud (2012) selon lesquels, un des aspects supposés influencer la qualité de l'information comptable est les caractéristiques internes de l'entreprise (l'organisation du service comptable pour notre cas) au sens ou la qualification du comptable, l'organisation et la digitalisation du service comptable devraient favoriser la pertinence de l'information comptable. Deux types de conclusions peuvent être tirés sur l'invalidation de H1.

Premièrement cela peut être dû à l'immaturité des services comptables des entreprises agricoles attendu que le secteur vient d'être introduit dans le système fiscal. Deuxièmement nous supposons que l'invalidation de cette hypothèse se justifie par le caractère saisonnier de l'activité agricole. En effet, le rythme discontinu de la production agricole conduit fréquemment à l'externalisation de la fonction comptabilité. Dès lors, la modestie (voir même l'absence) des structures comptables au sein des entreprises agricoles à contrarier notre appréciation de la contribution de l'organisation du service comptable à la détermination de la qualité de l'information comptable des entreprises relevant de notre échantillon.

\section{Conclusion}

Conscient de l'importance de l'utilité de l'information comptable dans le processus décisionnel dévoué aux choix d'investissement ou d'affectation des ressources, notre attirance se fut immédiatement sur les qualités qui rendent l'information comptable utile. Si bien qu'il a été assigné à ce modeste travail l'objectif d'apprécier l'impact de l'organisation du service comptable d'une entreprise sur la qualité de son information comptable (Azhari, 2021). 
Eu égard à la complexité et la rudesse de la tâche de l'opérationnalisation des questions liées à la qualité des divulgations comptable, et par souci de simplification, cet objectif global a été décliné en deux sousobjectifs. Pour ce qui est du premier sous-objectif, il consiste en une opérationnalisation originale du concept de la qualité de l'information comptable. Nous souhaitons contribuer, méthodologiquement et techniquement, à la mise en place d'une « métrique » de nature à apporter une valeur ajoutée à la quantification et l'évaluation de la qualité de l'information comptable qui demeure, à l'heure actuelle, un champ d'investigation tant actif que controversé. Dans le présent essai nous avons désigné la qualité de l'information comptable comme une variable endogène de troisième ordre que nous avons expliqué dans un premier temps par la dimension « organisation du service comptable » et en deuxième temps nous avons spécifié les trois variables du modèle comme des variables latentes de premier ordre associées directement à leurs blocs de mesure (Azhari, 2021).Pour ce qui est du deuxième sous-objectif, il a été nécessaire de motiver nos choix méthodologiques. À ce niveau de l'étude, nombreuses ont été les raisons qui appuyaient notre choix d'appréhender la réalité de notre recherche à travers une investigation par modélisation structurelle à variables latentes sous l'approche PLS (Sosik et al., 2009). Notre modèle hypothétique a été estimer selon une approche statistique de deuxième génération PSLPM ayant permis la validation des hypothèses y afférentes.

\section{Les implications de la recherche}

Certes que les recherches académiques dévouées aux questions liées à l'information comptable ont pris de l'ampleur ces dernières années. Hormis, le lecteur peut aisément repérer que ces études penchent particulièrement sur la perception de l'utilité ou de l'utilisation de l'information comptable. Cependant, l'analyse de la liaison entre l'organisation su service comptable et la qualité de l'information comptable reste un couloir peu fréquenté. Ceci dit, les implications de ce modeste travail s'observent à trois niveaux : théoriques, méthodologiques et managériaux.

\subsection{Les implications théoriques}

Les résultats étalés dans le présent travail favorisent un intérêt double : d'un côté ils cautionnent une substance empirique sur un angle peu abordé par la littérature qu'est les facteurs déterminants de la qualité de l'information comptable des entreprises. De l'autre côté ils se livrent à la problématique de la qualité de l'information comptable dans un contexte spécial qui est le secteur agricole dont le traitement comptable des actifs est inassimilable aux autres secteurs.

\subsection{Les implications méthodologiques}

L'une des contributions méthodologiques de ce travail été la confirmation notre intuition sur l'unidimensionnalité et la teneur théoriques des échelles de mesure que nous avons proposée et mobilisés. S'ajoute à cela l'utilisation d'une approche statistique de deuxième génération qui s'apparente aux modèles à équations structurelles (MES) à variable latente basées sur la méthode des moindres carrés partiels (MCP). 


\subsection{Les implications managériales}

Si l'on souhaite exposer l'intérêt principal qui en découle des implications managériales du présent travail, on peut citer la contribution de l'étude à l'assurance d'une compréhension meilleure de la qualité de l'information comptable des entreprises agricoles de la région Souss-Massa. C'est bien là un point considérable que nous pensons pouvoir contribuer par notre recherche. Dans le même sillage, cette recherche est motivée par la volonté d'assister les utilisateurs de l'information comptable des entreprises agricoles à distinguer les facteurs en situation de mettre en péril l'image fidèle du patrimoine et des résultats que les états de synthèses sont censés refléter.

\section{Les limites de la recherche}

La critique la plus vive que l'on peut adresser à notre positionnement théorique est inhérente à l'ancrage sur lequel gravite, dans la mesure où il est caractérisé par un développement théorique naissant et en perpétuelle évolution favorisant des échelles de mesure malingre. Bien que nous avons obtenu des résultats statistiquement très significatifs sur les qualités psychométriques des blocs de mesure retenus, notre choix de former la nouvelle variable latente composite (organisation du service comptable de l'entreprise) dépourvue d'une maturité théorique reste une limite pour notre modèle conceptuel. Du point de vue méthodologique, notre humble travail présente également certaines limites. La première se rattache à la composition de l'échantillon. En effet, la carence de données recensées des entreprises agricoles par les organismes a débouché sur une population indéfinie, dont la taille réelle et méconnue. Face à une telle situation, la détermination d'un échantillon probabiliste a été inconcevable, en conséquence nous nous sommes retournés vers un type d'échantillonnage non aléatoire (technique d'échantillonnage par convenance).

\section{Les voies futures de la recherche}

Une première piste prometteuse que nous prévoyons sonder consiste à un élargissement au niveau des variables latentes et des variables manifestes pour se remédier à la limite déjà évoquer dans ce sens. D'abord nous explorant un pan de la littérature en sciences de gestion qui s'intéresse à l'étude de degré de complication des systèmes d'information comptable dans les entreprises. Ce dernier associe la qualité de l'information comptable aux pratiques employer pour sa production. Plus précisément, une entreprise a plus de chance que sa qualité de l'information soit supérieure s'elle arrive à la produire dans des délais réduits avec des fréquences régulières. Ensuite, nous partant du développement de Chapellier (1977) qui incorpore la notion de l'utilité de l'information comme caractéristique essentielle à l'appréciation de sa qualité. De ce fait, l'auteur insiste sur le degré d'utilisation, par les dirigeants, de l'information comptable produite comme déterminante de la qualité de l'information comptable (Abdou et Dupuy, 1992). Pour finir, quelle que soit la méthode d'opérationnalisation choisie, un élargissement du champ spatiotemporel est subséquemment recommandé. Au niveau spatial l'analyse peut-être étalée sur l'échelle nationale. Sur le plan temporel des analyses longitudinales sur une longue période, à pas 
quinquennaux, apporteraient de plus amples éclairages sur les facteurs qui contribuent le plus à la détermination de la qualité de l'information comptable.

\section{BIBLIOGRAPHIE}

A

Abdoli, M. R., et Royaee, R. (2012). Board monitoring and earnings quality: an empirical study in Iran. African Journal of Business Management, 6(11), $4179-4184$.

Abdou, H. (1991). L'influence de l'évolution des systèmes de production sur le système d'information comptable: étude empirique (Doctoral dissertation, Montpellier 2).

Ahmed, A. S., et Duellman, S. (2007). Accounting conservatism and board of director characteristics: An empirical analysis. Journal of accounting and economics, 43(2-3), 411-437.

Aifuwa, H. O., et Embele, K. (2019). Board Characteristics and Financial Reporting. Journal of Accounting and Financial Management, 5(1), 30-44.

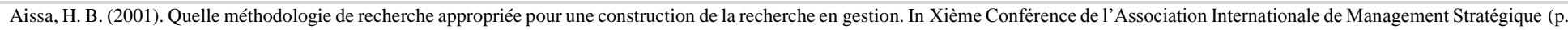
27)

Alkdai, H. H., et Hanefah, M. M. (2012). Audit committee characteristics and earnings management in Malaysian Shariah-compliant companies. Business and Management Review, 2(2), 52-61.

AZHARI A. (2021). Essai d'analyse des déterminants de la qualité de l'information comptable des entreprises agricoles : Cas de la région Souss-Massa. (thèse)

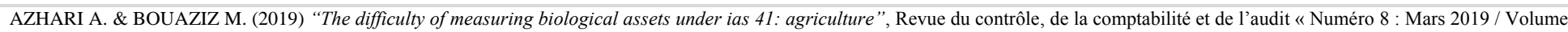
$3:$ numéro $4 » \mathrm{p}: 29-41$

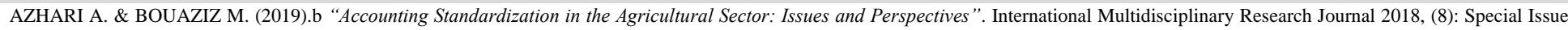
01-17 doi: 10.25081/imrj.2018.v8.3674.

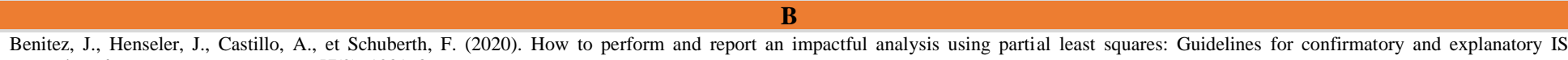

\begin{tabular}{l} 
research. Information et Management, 57(2), 103168. \\
\hline Bushman, R. M., et Smith, A. J. (2001). Financial accounting information and corporate governance. Journal of accounting and Economics, 32(1-3), 237-333.
\end{tabular}

Carricano, M., Poujol, F., et Bertrandias, L. (2010). Analyse de données avec SPSS®. Pearson Education France.

Chapellier, P. (1997). Profils de dirigeants et données comptables de gestion en PME. Revue internationale PME Économie et gestion de la petite et moyenne entreprise, 10(1), 9-41.

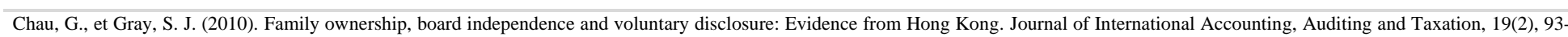
109.

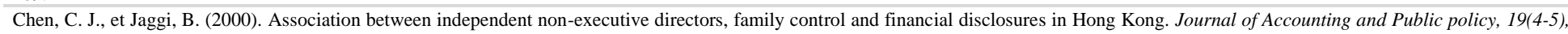
$285-310$.

Chin, W. W. (1998). Commentary: Issues and opinion on structural equation modeling.

Chin, W. W. (1998). The partial least squares approach to structural equation modeling. Modern methods for business research, 295(2), 295-336.

Coase, R. H., Gillis, X., et Bourreau, M. (1987). La nature de la firme. Revue française d'économie, 2(1), 133-163.

Courpasson, D. (1997). Régulation et gouvernement des organisations. Pour une sociologie de l'action managériale. Sociologie du travail, 39-61.

Cuijpers, R., et Buijink, W. (2005). Voluntary adoption of non-local GAAP in the European Union: A study of determinants and consequences. European accounting review, 14(3), 487-524.

\section{D}

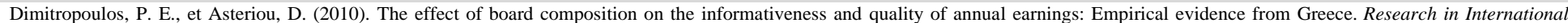
Business and Finance, 24(2), 190-205.

E

Eng, L. L., \& Mak, Y. T. (2003). Corporate governance and voluntary disclosure. Journal of accounting and public policy, 22(4), 325-345.

\section{F}

Fama, E. F., \& Jensen, M. C. (1983). Separation of ownership and control. The journal of law and Economics, 26(2), 301-325.

Fathi, J. (2013). Corporate governance system and quality of financial information. Mediterranean Journal of Social Sciences, 4(2), 129.

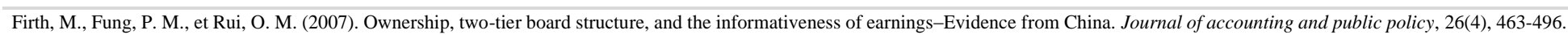

Fornell, C. (1982). A second generation of multivariate analysis. Measurement and evaluation (Vol. 2). Praeger Publishers.

Fornell, C., et Bookstein, F. L. (1982). Two structural equation models: LISREL and PLS applied to consumer exit-voice theory. Journal of Marketing research, 19(4), 440-452.

\section{G}

Garson, G. D. (2016). Partial least squares: Regression and structural equation models. Asheboro, NC: Statistical Associates Publishers.

Geisser, S. (1975). The predictive sample reuse method with applications. Journal of the American statistical Association, 70(350), 320-328.

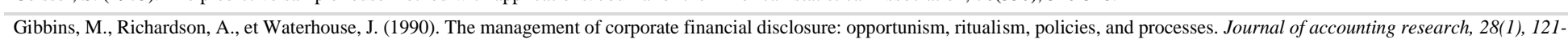
143. 


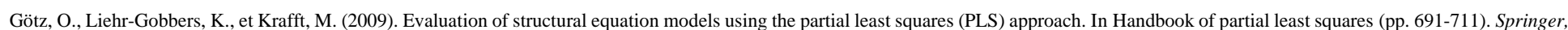
Berlin, Heidelberg.

Gupta, S. D., \& Ray, D. S. (1988). Optical multistability in a nonlinear Fibonacci multilayer. Physical Review B, $38(5), 3628$.

H

Habib, A., et Azim, I. (2008). Corporate governance and the value-relevance of accounting information: Evidence from Australia. Accounting Research Journal, 21(2), 167-194.

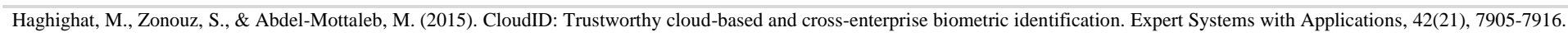

Hair Jr, J. F., Hult, G. T. M., Ringle, C., et Sarstedt, M. (2016). A primer on partial least squares structural equation modeling (PLS-SEM). Sage publications.

Hair Jr, J. F., Sarstedt, M., Hopkins, L., et Kuppelwieser, V. G. (2014). Partial least squares structural equation modeling (PLS-SEM). European business review.

Han, S. (2004). Ownership structure and quality of financial reporting. Available at SSRN 591801.

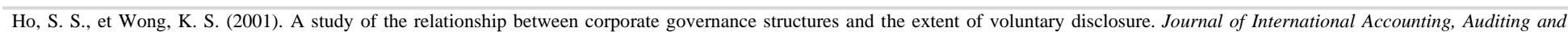
Taxation, 10(2), 139-156.

Holmes, S., et Nicholls, D. (1989). Modelling the accounting information requirements of small businesses. Accounting and Business Research, 19(74), 143-150.

Holtz, L., et Sarlo Neto, A. (2014). Effects of board of directors' characteristics on the quality of accounting information in Brazil. Revista Contabilidade et Finanças, 25(66), 255-266.

Hopwood, A. G. (1987). The archeology of accounting systems. Accounting, organizations and society, 12(3), $207-234$.

Huafang, X., \& Jianguo, Y. (2007). Ownership structure, board composition and corporate voluntary disclosure: Evidence from listed companies in China. Managerial auditing journal.

\section{J}

Jensen, M. C., et Meckling, W. H. (1976). Theory of the firm: Managerial behavior, agency costs and ownership structure. Journal of financial economics, 3(4), 305-360.

John, K., et Senbet, L. W. (1998). Corporate governance and board effectiveness. Journal of banking et Finance, 22(4), 371-403.

\section{L}

Lara, J. M. G., Osma, B. G., et Penalva, F. (2009). Accounting conservatism and corporate governance. Review of accounting studies, 14(1), 161-201.ISO 690

Leung, S., \& Horwitz, B. (2004). Director ownership and voluntary segment disclosure: Hong Kong evidence. Journal of International Financial Management \& Accounting, 15(3), 235-260.

\section{M}

Marra, A., Mazzola, P., et Prencipe, A. (2011). Board monitoring and earnings management pre-and post-IFRS. The International Journal of Accounting, 46(2), 205-230.

Mezias, S. J. (1990). An institutional model of organizational practice: Financial reporting at the Fortune 200. Administrative science quarterly, $431-457$.

Michailesco, C. (1998). Contribution à l'étude des déterminants de la qualité de l'information comptable diffusée par les entreprises françaises (Doctoral dissertation, Paris 9).

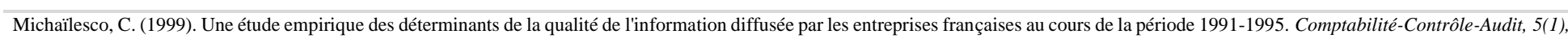
pp-83.

Michaïlesco, C. (2000). Perspectives pour la recherche sur les déterminants de l'offre d'information des entreprises. Comptabilité-Contrôle-Audit, 6(3), 83-94.

Miles, S., et Miles, S. (2019). Stakeholder Theory and Accounting. The Cambridge Handbook of Stakeholder Theory, 173.

Nagar, V. (1999). The role of the manager's human capital in discretionary disclosure. Journal of Accounting Research, 37, $167-181$.

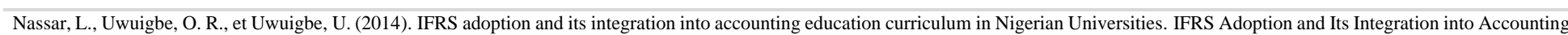
Education Curriculum in Nigerian Universities.

Norita, M. N., \& Shamsul-Nahar, A. (2004). Voluntary disclosure and corporate governance among distressed firms in Malaysia. Financial Reporting, Regulation and Governance, 3(1).

\section{O}

O'sullivan, N. (2000). The impact of board composition and ownership on audit quality: evidence from large UK companies. The British Accounting Review, 32(4), $397-414$.

R

Raffournier B. (1997). « The determinants of voluntary financial disclosure by Swiss listed companies: a reply », The European Accounting Review, vol. 6, ${ }^{\circ} 3$, pp.493-96

Ringle, C. M., Sarstedt, M., et Straub, D. W. (2012). Editor's Comments: A Critical Look at the Use of PLS-SEM in" MIS Quarterly". MIS quarterly, iii-xiv.

\section{S}

Sardeshmukh, S. R., et Vandenberg, R. J. (2017). Integrating moderation and mediation: A structural equation modeling approach. Organizational Research Methods, 20(4), 721-745.

Sarstedt, M., Ringle, C. M., et Hair, J. F. (2017). Partial least squares structural equation modeling. Handbook of market research, 26, 1-4

Scott, W. R. (1977). Effectiveness of organizational effectiveness studies. New perspectives on organizational effectiveness, 63-95.

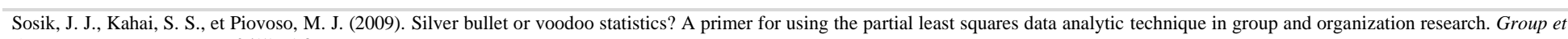
Organization Management, 34(1), 5-36.

Stiglitz, J. E., Walsh, C. E., Lafay, J. D., \& Mayer, F. (2000). Principes d'économie moderne. de Boeck.

Stone, M. (1974). Cross-validation and multinomial prediction. Biometrika, 61(3), 509-515. 
Tenenhaus, M., Vinzi, V. E., Chatelin, Y. M., et Lauro, C. (2005). PLS path modeling. Computational statistics et data analysis, 48(1), $159-205$.

Vafeas, N. (2000). Board structure and the informativeness of earnings. Journal of Accounting and Public policy, 19(2), 139-160.

Verrecchia, R. E. (2001). Essays on disclosure. Journal of accounting and economics, 32(1-3), 97-180.

Wacheux, F. (1996). Méthodes qualitatives et recherche en gestion. Economica. 